F. UPPER ATMOSPHERES 


\title{
THE EFFECT OF ATMOSPHERIC DYNAMICS ON THE UPPER ATMOSPHERE PHENOMENA OF MARS AND VENUS
}

\author{
MIKIO SHIMIZU \\ Dept. of Physics, Ochanomizu University, Tokyo, Japan and Institute of Space and Aeronautical \\ Science, University of Tokyo, Komaba, Meguro-ku, Tokyo, Japan
}

\begin{abstract}
By the observations of Mariners and Veneras, oxygen atom deficiencies and very low exospheric temperatures were found in the upper atmospheres of Mars and Venus. There are two types of interpretations for these phenomena: photochemical ones $\left(\mathrm{CO}_{3}\right.$ hypothesis), and dynamical ones. In this paper, the latter point of view is summarized. It was emphasized that the effect of atmospheric mixing due to general circulation and/or eddy diffusion is important for the understanding of the Cytherean and Martian atmospheric phenomena.
\end{abstract}

A series of 'Mariner' space probes measured the electron densities in the Martian and Cytherean ionospheres by using the S-band radio occultation technique and found that the altitudes of the peaks of the density profiles were very low and that the widths of the profiles were very narrow. The characteristics may be correlated with the fact that these atmospheres are mainly composed of $\mathrm{CO}_{2}$. After the success of Mariner 4 observation, $\mathrm{E}, \mathrm{F}_{1}$, and $\mathrm{F}_{2}$ models have been presented to interpret these characteristics. Recently, however, the students in this field have reached a general agreement that the ionospheres are of the $\mathrm{F}_{1}$-layer type (the observed temperature profiles in the lower atmospheres are too high for the $F_{2}$-layer model, and the E-layer model has difficulty in explaining the non-existence of the $F_{1}$ layers).

If these ionospheres are of $F_{1}$-layer type, the amounts of oxygen atoms in the upper atmospheres of these planets should be so small as to depress their $F_{2}$ layers, yet it is certain that $\mathrm{CO}_{2}$ is dissociated to $\mathrm{CO}$ and $\mathrm{O}$ by Schumann-Runge radiation. Therefore the whereabouts of $\mathrm{O}$ atoms on these planets raises a new question. The deficiency of $\mathrm{O}$ atoms may be approached from two standpoints: the photochemical explanation and the dynamical one. Recently McElroy and Hunten (1970) speculated that $O\left({ }^{1} D\right)$ from the dissociation of $\mathrm{CO}_{2}$ reacts with $\mathrm{CO}_{2}$ to make $\mathrm{CO}_{3}^{*}$ and that this activated complex might easily be deactivated to its ground state by emitting photons. Then $\mathrm{CO}_{3}$ may react with $\mathrm{CO}$ to make $2 \mathrm{CO}_{2}$ and the overall process result in a very rapid recombination of $\mathrm{CO}$ and $\mathrm{O}$. $\mathrm{CO}_{3}^{*}$ may, however, be much more rapidly decomposed to $\mathrm{CO}_{2}$ and $\mathrm{O}\left({ }^{3} P\right)$. In other words, the reaction between $\mathrm{O}\left({ }^{1} \mathrm{D}\right)$ and $\mathrm{CO}_{2}$ may be of the pre-dissociation type, as in the case of $\mathrm{O}\left({ }^{1} D\right)$ and $\mathrm{N}_{2}$; therefore the $\mathrm{CO}_{3}$ explanation may not work in planetary upper atmospheres.

This paper presents an alternate, dynamical, interpretation for the O-atom deficiency: the dilution of the dissociation products in the upper atmosphere by atmospheric mixing. In order to discuss a problem of this type, we must solve a set of equations of continuity and motion simultaneously. The coupling between these equations is in particular strong in the case of the dissociation of the major atmospheric component. (It should be noted that the flux equation used by McElroy and Hunten - Equation 
(3) of $\S 3$ in their paper - can apply only to the case of dissociation of a minor component.) We have already solved this problem under proper boundary conditions (Shimazaki and Shimizu, 1970; Shimizu, 1969a). Here the physical implication of the results will be explained schematically.

At first we shall discuss the upper atmospheric phenomena by taking into account the photochemical processes and the effect of molecular diffusion, and by neglecting the effect of turbulence. The dissociation cross-section of $\mathrm{CO}_{2}$ is much smaller than that of $\mathrm{O}_{2}$. Thus the Schumann-Runge radiation penetrates deeper into the atmosphere in the case of Mars and Venus than it does on earth. The upper part of Figure 1 describes this situation. It is noteworthy that there is a subtle difference between Mars and Venus, because of the difference of their distances from the sun. The effect of molecular diffusion decreases the densities of the dissociation products on Mars, while it has no influence on Venus.

Next we shall take the effect of turbulence into account. The planetary atmospheres

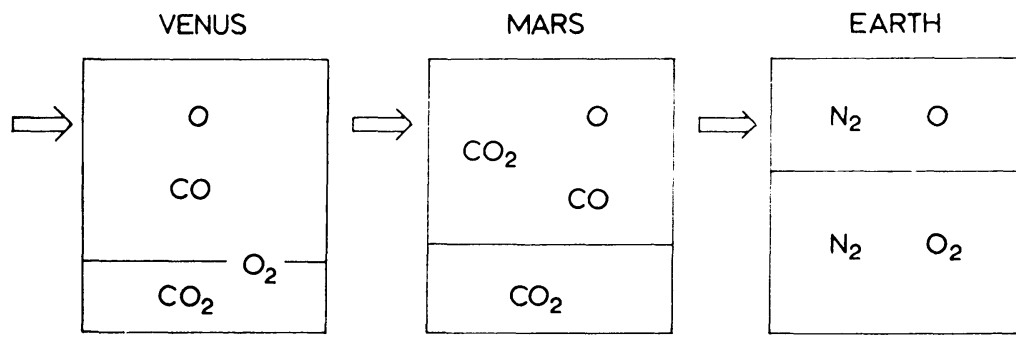

NEUTRAL ATMOSPHERIC COMPONENT
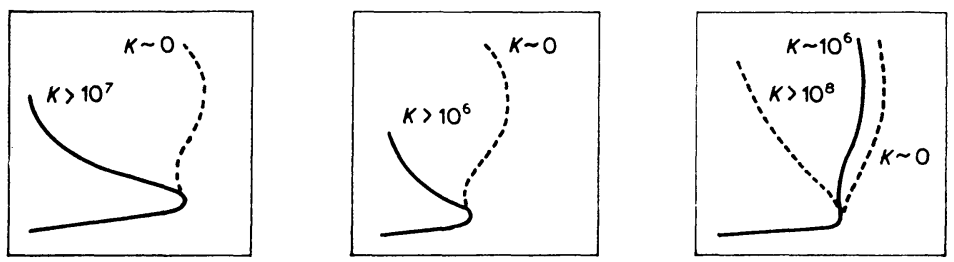

ELECTRON PROFILE

Fig. 1. A comparison of upper atmospheres and ionospheres of Venus, Mars, and Earth. In the upper part, the distributions of atmospheric constituents in the upper atmospheres of the planets are schematically shown (see text). In the lower part, the calculated electron density profiles for various values of $\kappa$, eddy diffusivity, are shown. The bold lines correspond to the observational curves.

are usually well mixed under their turbopauses, the positions of which are marked by the horizontal arrows in Figure 1. It may be intuitively clear that the effect of turbulence works more efficiently on Mars and Venus than on Earth. In the lower part of Figure 1, we plotted the calculated electron density profiles for various eddy diffusion coefficients $\kappa$ on each planet. In the case of Earth, an $\mathrm{F}_{2}$ layer exists even in the case of $\kappa=10^{6} \mathrm{~cm}^{2} / \mathrm{sec}$. In order to depress the $\mathrm{F}_{2}$ layer, a large value of $\kappa, 10^{8} \mathrm{~cm}^{2} / \mathrm{sec}$, is required. On the other hand, $\kappa$ of the order of $10^{6}$ 
$\mathrm{cm}^{2} / \mathrm{sec}$ is enough to depress the $\mathrm{F}_{2}$ layer on Mars. In the case of Venus, $10^{7}$ $\mathrm{cm}^{2} / \mathrm{sec}$ is necessary for $\kappa$, because the dissociation of $\mathrm{CO}_{2}$ is more complete on this planet.

In the case of Mars, $\mathrm{O}\left({ }^{3} P\right)$ and $\mathrm{CO}$ may be transported downwards to the level where the time constants of mixing and recombination become nearly equal (Figure 2).

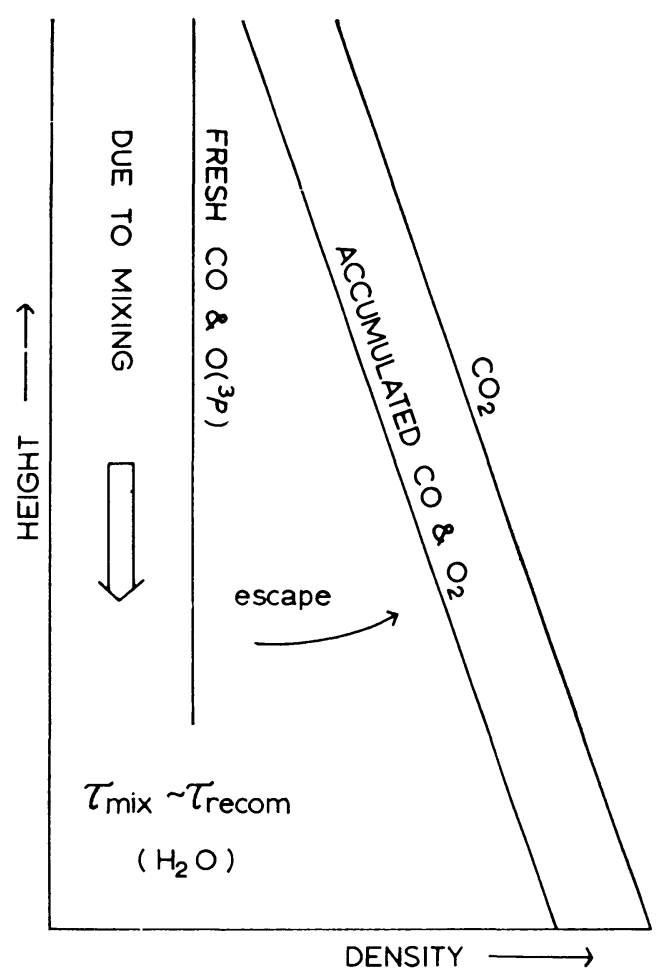

Fig. 2. Recombination and accumulation mechanism of $\mathrm{CO}$ in the lower atmosphere of Mars.

At this level they will be almost completely recombined back to $\mathrm{CO}_{2}$ by the catalytic reaction with $\mathrm{H}_{2} \mathrm{O}$ (Reeves et al., 1966). This reaction works only in the lower atmosphere where the atmospheric density is high. A very small production of $\mathrm{O}_{2}$ and $\mathrm{CO}$ may be expected, and they will accumulate during a long time. As for Venus, the high temperature of its lower atmosphere may play a role to keep the concentration of $\mathrm{O}_{2}$ and $\mathrm{CO}$ at the thermochemical equilibrium values.

Now, we shall consider another evidence for a strong dynamical effect in the upper atmosphere of Mars. From the electron density profile obtained by Mariner 4, a very low plasma temperature in the Martian ionosphere, $300 \mathrm{~K}$, was obtained. The exospheric temperature, $T_{\text {exo }}$, can be a little lower than this value. McElroy (1969) calculated $T_{\text {exo }}$ by taking into account the cooling of the atmosphere due to $\mathrm{CO}_{2}$ infrared emission near the mesopause; the cooling causes a steep temperature gradient between the exosphere and mesopause. The heat deposited at the exosphere may be transported 
to the lower region by molecular heat conduction. Then $T_{\text {exo }}$ may be decreased (Figure 3). McElroy obtained a value however, of about $500 \mathrm{~K}$, much higher than the observed one. As the mesopause temperature was already low enough, it was in principle difficult to decrease $T_{\text {exo }}$ less than this value by this mechanism. We think that one way to solve this puzzle is to increase the conductivity between these regions by taking into account the heat transport due to turbulence.

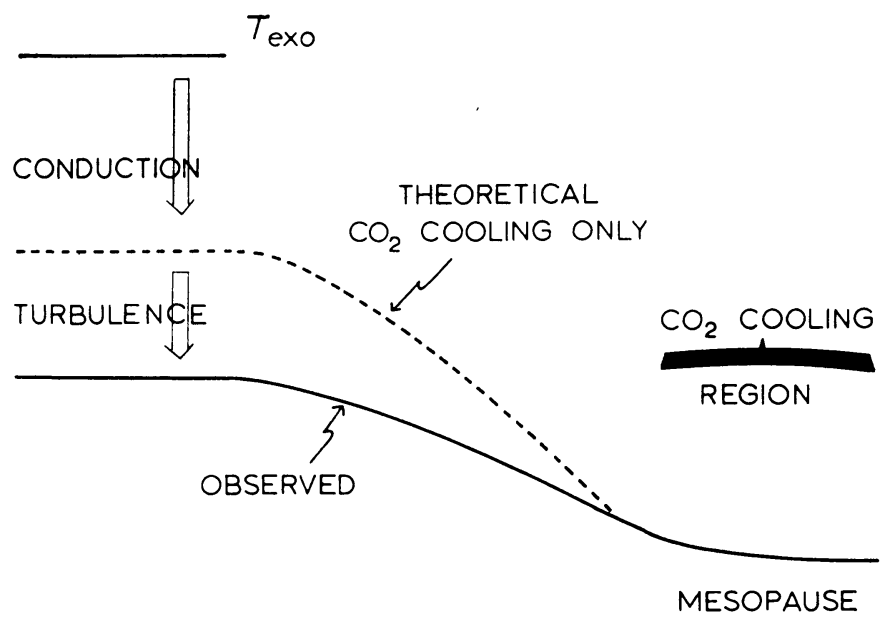

Fig. 3. Explanation for the low exospheric temperature of Mars.

At this symposium Kliore suggested plasma temperatures around $450 \mathrm{~K}$ at the time of Mariner 6 and 7. The $T_{\text {exo }}$ derived from the ultraviolet measurement (probably of $\mathrm{O}$ atoms) appears to suggest a similar low value. But for this time the calculated value of McElroy was reported as $700 \mathrm{~K}$, again much higher than observed. Such a situation was already predicted in our paper (Shimizu, 1969b) before the success of Mariner 6 and 7. By taking into account the heat transport by the eddy diffusion, we have solved the heat balance equation. By comparing the results with the new data presented in this symposium, we estimate the value of the eddy diffusivity $\kappa$ in the Martian upper atmosphere as $7-10 \times 10^{7} \mathrm{~cm}^{2} / \mathrm{sec}$. Horizontal atmospheric mixing due to a wind in the upper atmosphere of the velocity of several hundred kilometers/sec can equivalently explain the low exospheric temperature (see Shimizu, 1969a).

Gierasch and Goody (1968) derived theoretically so high an eddy diffusivity in the lower atmosphere of Mars as $10^{8} \mathrm{~cm}^{2} / \mathrm{sec}$. The internal gravity waves from this region may go up to the upper atmosphere and will dissipate there by interaction with the wind shear. It has already been suggested by Goody and Belton (1967) that the Martian upper atmosphere can easily be turbulent due to radiative instability. Thus strong turbulence with $\kappa \sim 10^{8} \mathrm{~cm}^{2} / \mathrm{sec}$ may be expected in the upper atmosphere. It may be argued that the temperature near mesopause may be a little increased by this forced mixing effect. The $\mathrm{CO}_{2}$ cooling mechanism works, however, more effectively as the 
temperature increases. Therefore, it can be a good thermostat for the mesopause temperature.

In the Cytherean case, two different $T_{\text {exo }}$ values have been presented. From the deuterium model (Barth, 1968), it might be $650 \mathrm{~K}$. If we take, however, the hot-atom model (Barth, this symposium), it should be $325 \mathrm{~K}$. At first we shall assume that $T_{\text {exo }}$ is $650 \mathrm{~K}$. This value is in good agreement with the calculated value by McElroy (1969) for a pure $\mathrm{CO}_{2}$ atmosphere. (This does not mean that the $\mathrm{CO}_{3}$ scheme works; a strong mixing effect can also explain the apparent depression of $\mathrm{CO}_{2}$ dissociation.) This suggests that $\kappa$ may be less than $10^{7} \mathrm{~cm}^{2} / \mathrm{sec}$ on Venus, because in this case the time constant of the mixing effect is longer than that of the $\mathrm{CO}_{2}$ cooling effect and we can expect no effect of turbulence or general circulation on $T_{\text {exo }}$. Therefore, together with the result of the discussion on the Cytherean ionosphere, we may conclude that the value for $\kappa$ on the Venusian upper atmosphere is about $1 \times 10^{7} \mathrm{~cm}^{2} / \mathrm{sec}$. (Of course, wind with a velocity of $100 \mathrm{~m} / \mathrm{sec}$ has a similar effect.) If the lower value reported by Barth in this symposium is right, however, the situation may be changed and we must assume a stronger wind or a larger eddy diffusivity in the upper atmosphere of Venus.

In summary, we have explained many observational facts in the upper atmospheres of Mars and Venus, by using only one parameter $\kappa$. Without the recognition of the strong dynamical effect, we might adopt many artificial assumptions. For instance, McElroy and Hunten assumed a speculative $\mathrm{CO}_{3}$ scheme. Then the formation of $\mathrm{O}_{2}$ and $\mathrm{CO}$ will be forbidden in the upper atmosphere. Therefore they could not help assuming very complex chemical processes to explain the existence of $\mathrm{CO}$ in the Martian atmosphere. Furthermore, in order to resolve the discrepancy in the Martian exospheric temperature, Cloutier et al. (1969) speculated that the solar wind might change the electron density profile of Mars. The reason why the same solar wind fails to affect the Cytherean ionosphere is not yet clearly explained. Furthermore, they neglected an important effect, the rotation of Mars, in their discussion.

Finally we hope to remark here that we have already obtained the result that a similar type of strong dynamical effect may exist in the Jovian upper atmosphere as well as in the Martian and Cytherean ones (Shimizu, 1970).

Note added in proof: Recently, Slanger and Black confirmed directly by resonance fluorescent technique that the quantum yield of $\mathrm{O}\left(\mathrm{P}^{3}\right)$ in $\mathrm{CO}_{2}$ photolysis is unity due to very rapid deactivation. Shimizu showed that the wind due to solar EUV differential heating decreased $T_{\text {exo }}$ of Venus and Mars effectively (ISAS report, University of Tokyo, Nos. 455 and 456.

\section{References}

Barth, C. A.: 1968, 'Interpretation of the Mariner 5 Lyman Alpha Measurements', J. Atmospheric Sci. 25, 564.

Cloutier, P. et al.: 1969, 'Modification of the Martian Ionosphere by the Solar Wind', J. Geophys. Res.

Gierasch, P. and Goody, R.: 1968, 'A Study of the Thermal and Dynamical Structure of Martian Lower Atmosphere', Planetary' Space Sci. 16, 615.

23-P.A. 
Goody, R. and Belton, M. J. S.: 1967, 'Radiative Relaxation Times on Mars', Planetary Space Sci. 15, 247.

McElroy, M. B.: 1969, 'Structure of Venus and Mars Atmospheres', J. Geophys. Res. 74, 29.

McElroy, M. B. and Hunten, D. M.: 1970, 'The Photochemistry of $\mathrm{CO}_{2}$ in the Atmosphere of Mars', to appear.

Reeves, R. R. et al.: 1966, 'Photochemical Equilibrium Studies of Carbon Dioxide and Their Significance for the Venus Atmosphere', J. Phys. Chem. 70, 1637.

Shimazaki, T. and Shimizu, M.: 1970, 'Model Calculation of the Martian Upper Atmosphere and Ionosphere with Transport Effects', Rept. Ionos. Space Res. Japan 24, 80.

Shimizu, M.: 1969a, 'A Model Calculation of the Cytherean Upper Atmosphere', Icarus 10, 11.

Shimizu, M.: 1969b, 'Atmospheric Structures of Planets', Ōyo-butsuri 38, 284 (in Japanese).

Shimizu, M.: 1970, 'The Upper Atmosphere of Jupiter: Dissociation and Ionization of Hydrogen' Icarus. 